\title{
The role of contrast-enhanced ultrasound in neurosurgical disease
}

\author{
Kathryn N. Kearns, BS, ${ }^{1}$ Jennifer D. Sokolowski, MD, PhD, ${ }^{1}$ Kimberly Chadwell, MPH, ACS, RDCS, ${ }^{1}$ \\ Maureen Chandler, RDMS, RVT, RT(R), ${ }^{1}$ Therese Kiernan, AS, RDMS, RVT, ${ }^{1}$ \\ Francesco Prada, MD, ${ }^{1,2}$ M. Yashar S. Kalani, MD, PhD, ${ }^{1}$ and Min S. Park, MD ${ }^{1}$
}

1Department of Neurological Surgery, University of Virginia Health System, Charlottesville, Virginia; and 2Department of Neurosurgery, Fondazione IRCCS Istituto Neurologico C. Besta, Milan, Italy

Contrast-enhanced ultrasound (CEUS) is a relatively new imaging modality in the realm of neurosurgical disease. CEUS permits the examination of blood flow through arteries, veins, and capillaries via intravascular contrast agents and allows vascular architectural mapping with extreme sensitivity and specificity. While it has established utility in other organ systems such as the liver and kidneys, CEUS has not been studied extensively in the brain. This report presents a review of the literature on the neurosurgical applications of CEUS and provides an outline of the imaging modality's role in the diagnosis, evaluation, and treatment of neurosurgical disease.

https://thejns.org/doi/abs/10.3171/2019.9.FOCUS19624

KEYWORDS contrast-enhanced ultrasound; imaging; neurosurgery

$\mathrm{C}$ ONTRAST-ENHANCED ultrasound (CEUS) involves the use of an intravascular contrast agent to visualize arterial, capillary, and venous blood flow while reducing signal from the adjacent brain parenchyma.,23,29 Unlike agents used in other imaging techniques, CEUS contrast agents consist of microbubbles approximately 5 $\mu \mathrm{m}$ in diameter that do not diffuse into the surrounding parenchyma. ${ }^{4,6,21,24,25}$ These microbubbles consist of a phospholipid outer layer that ensures the contrast remains in the intravascular space. ${ }^{6,11,21}$ This allows for sharp and accurate visualization of the vessel lumen. Contrast can be administered through a peripheral vein to evaluate the four vascular phases: arterial, peak, parenchymal, and venous. ${ }^{6,21,23}$ Given that the contrast passes through lung capillaries, the arterial system can be visualized with venous administration of the agent. ${ }^{25}$ Vascular information can be gleaned from the direction and velocity at which the microbubbles flow through circulation as well as the enhancement intensity of the contrast agent at specific times after administration. ${ }^{23}$ Specific harmonic waves are generated when mi- crobubbles interact with low mechanical index ultrasound; harmonic signals are then reflected back to the probe and managed by contrast-specific algorithms. ${ }^{21,25}$ Brain perfusion and parenchymal anatomy are measured using an algorithm that measures the echo intensity of these reflected harmonics over time. ${ }^{21,23}$ This algorithm suppresses linear ultrasound echogenicity of the surrounding parenchyma, isolating the nonlinear echogenicity of blood flow and allowing for visualization of the vascular architecture. ${ }^{6}$ In this article, we outline the advantages of CEUS in several categories of neurosurgical disease.

\section{Preliminary Experiences in Brain Tumors}

CEUS can provide invaluable information on solid intracranial tumors, particularly when combined with Doppler ultrasound. Kanno et al. examined the utility of intraoperative contrast-enhanced power Doppler ultrasound in a wide spectrum of intracranial tumors. ${ }^{17}$ They found that intraoperative knowledge of the vascular layout allows sur-

ABBREVIATIONS AVM = arteriovenous malformation; CEUS = contrast-enhanced ultrasound; DAVF = dural arteriovenous fistula; EFSUMB = European Federation of Societies for Ultrasound in Medicine and Biology; GBM = glioblastoma.

SUBMITTED July 29, 2019. ACCEPTED September 5, 2019.

INCLUDE WHEN CITING DOI: 10.3171/2019.9.FOCUS19624. 
geons to identify the safest route to approach deep-seated tumors before disrupting normal tissue. Surgical navigation was improved using this technique given increased knowledge of the location and architecture of peritumoral and intratumoral vessels. This was particularly useful in highly vascular lesions such as glioblastomas (GBMs), hemangioblastomas, meningiomas, and brain metastases. Prada and colleagues assessed CEUS in comparison to Gd-enhanced MRI in the visualization of high-grade GBM and in vessel localization in skull base tumors in particular. ${ }^{21,26}$ Using fusion imaging between preoperative MRI and real-time CEUS, these authors proved the capability of the latter modality to highlight the same target volume shown by preoperative Gd-enhanced MRI in patients with GBM (Fig. 1). Additionally, they reported its utility in modeling the $3 \mathrm{D}$ vascular structure in conjunction with information from preoperative MRI, which clarified the ideal surgical route in complicated approaches such as the frontolateral and retrosigmoid approaches. Prada et al. also found that precise tumor demarcation is more difficult to achieve in lower-grade gliomas, though borders are frequently visualized to an extent that nonetheless permits complete lesion resection. ${ }^{25}$ They evaluated several types of pathological intracranial structures and found that CEUS was unable to characterize abscesses or areas of radionecrosis. ${ }^{25}$ However, abscesses and radiation necrosis are highlighted by Gd-enhanced MRI, so combining imaging modalities can be helpful in differentiating these structures from other intracranial lesions.

Similarly, CEUS and color Doppler ultrasound can be used together for intraoperative embolization of highly vascular tumors. Della Pepa et al. examined the combined utility of CEUS and color Doppler ultrasound in the embolization of a posterior cranial fossa hemangioblastoma that did not permit preoperative endovascular evaluation. ${ }^{7}$ These combined techniques allowed the surgeons to guide direct puncture of the tumor for injection of the embolizing agent and also provided real-time information about the distribution of the agent and the subsequent change in blood flow through the area of interest. The contrast agent microbubbles concentrated in areas of increased vascularization and indicated the degree of blood supply in various areas within the tumor bulk.?

CEUS also has potential as a way to preoperatively identify tumor grade. ${ }^{5,6,17}$ Contrast enhancement has been shown to positively correlate with the angiographic grade of gliomas and hemangioblastomas. ${ }^{17}$ Microvessel density is increased in intracranial tumors and can reflect tumor progression and angiogenesis, which are indicative of overall tumor malignancy. ${ }^{5}$ Benign tumors have lower microvessel densities than malignant tumors and thus can be identified by observing the duration of contrast enhancement using the CEUS technique. Ellegala et al. used microbubble contrast targeted to $\alpha_{\mathrm{v}} \beta_{3}$ integrins, which are preferentially expressed on neovascular endothelium, to evaluate early glioma angiogenesis in a murine model. ${ }^{10}$ They found that signals from this targeted contrast agent were concentrated around the periphery of tumors, indicating microscopic progression of tumor vasculature that suggested overall growth of the tumor itself. Using nontargeted microbubbles, they also identified a correlation between slower visualized blood velocity and increased microvascular blood volume within and around gliomas. These authors monitored tumor growth over a 28-day period and found that as gliomas expand, angiogenesis progresses rapidly, though overall blood velocity decreases. Both of these characteristics can be identified with targeted CEUS techniques. This application of CEUS can be utilized to predict metastatic potential and overall prognosis of gliomas and other cerebral tumors, as neovascular abnormalities and vessel permeability are thought to contribute to tumor cell migration through the vasculature. ${ }^{10}$

A similar study on the use of CEUS in glioma characterization demonstrated two distinct patterns of contrast enhancement in GBM. ${ }^{6}$ The first pattern showed heterogeneous enhancement with scattered spots of increased enhancement between lower enhancing areas of necrotic tissue, while the second pattern exhibited a circumferential rim of enhancement around a nonenhancing necrotic core. ${ }^{6}$ Various flow patterns were identified that consistently indicated gliomas of specific grades and subcategories. For example, astrocytomas showed organized flow of the contrast agent through the feeding arteries and diffuse, less clearly defined venous drainage, whereas oligodendrogliomas exhibited faster contrast enhancement in both the arterial and venous phases. Some researchers were consistently able to identify a clearly defined border between tumor and normal parenchyma among various grades and enhancement patterns that contributed to more complete resection. 6,12,27 However, another glioma study reported conflicting results, suggesting that lower-grade gliomas have more irregular and less well-defined borders than higher-grade gliomas. ${ }^{5}$ Despite this, borders of lowgrade gliomas were still more visible with CEUS evaluation than with regular ultrasound, and complete resection was facilitated. ${ }^{5}$

The use of repeat intraoperative imaging leads to higher rates of complete lesion resection, as peritumoral contrast-

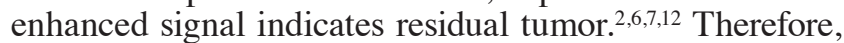
CEUS in solid tumor resection can be used preoperatively to identify a safe approach, intraoperatively to evaluate vascular migration as tumor debulking progresses, and after lesion resection to confirm complete excision. To this end, the use of CEUS has been added to the European Federation of Societies for Ultrasound in Medicine and Biology (EFSUMB) guidelines for neuro-oncological procedures. $^{29}$

\section{Vascular Abnormalities}

CEUS has intrinsic advantages when visualizing the organ vasculature as it allows the surgeon to visualize in real-time both high-flow and low-flow vessels as well as large- and small-caliber vessels simultaneously, independently from the angle of insonation. When CEUS is coupled with B-mode ultrasonography, surgeons can visualize the lesion, the feeding and draining vasculature, and changes in the surrounding anatomy attributable to mass effect. ${ }^{23,33}$ CEUS is notably useful in identifying the primary feeding artery and any concealed vasculature within dural arteriovenous fistulas (DAVFs). In a recent study, Prada et al. evaluated the role of CEUS in spinal DAVF surgery. They reported that CEUS allows visualization of 

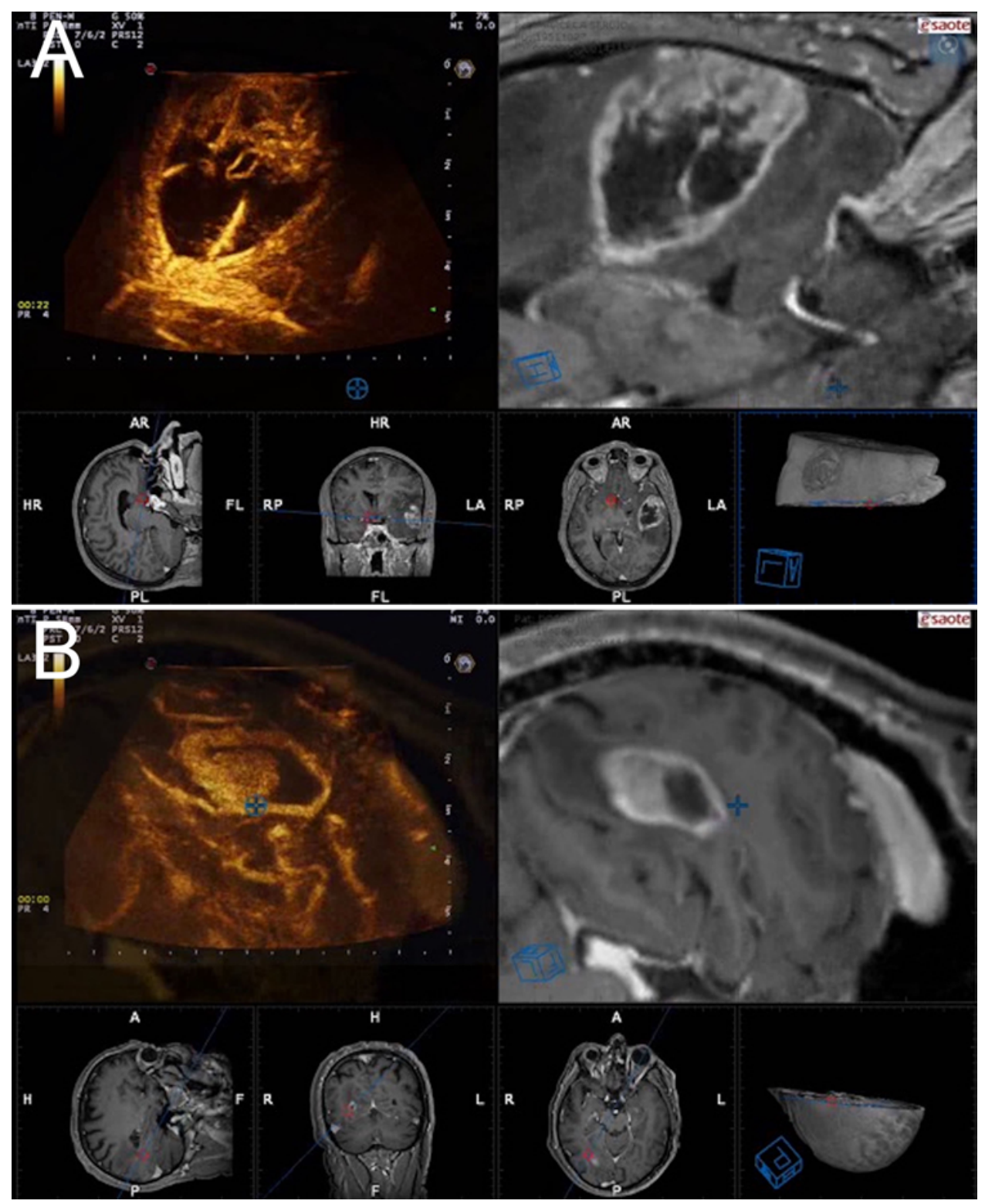

FIG. 1. Fusion imaging between CEUS (left upper) and Gd-enhanced T1-weighted MRI (right upper) in an axial view obtained through a temporal craniotomy. Acquisition plane (blue line) in operative sagittal, coronal, and axial projections and 3D reconstruction (lower left to right) in a case of temporal lobe GBM (A) and occipital lobe GBM (B) prior to resection.

the fistula before disrupting the dura mater, which reduces dural damage and results in more precise excision..$^{22}$ This can be particularly useful in evaluating deep-seated lesions. ${ }^{33} \mathrm{Xu}$ et al. reported the significant benefit of CEUS in the determination of intraoperative migration of vessels during resection of deep-seated arteriovenous malformations (AVMs). ${ }^{33}$ As surgery progresses, the vasculature and brain parenchyma shift with pressure changes and lesion debulking. This disrupts the vascular architecture identified with preoperative imaging. Intraoperative CEUS allows surgeons to distinguish feeding arteries and the AVM nidus from normal vessels in the evaluation of collateral circulation during the procedure. ${ }^{13,24,33}$

CEUS also has reported utility in evaluating aneurysmal and parenchymal flow after aneurysm clipping: Mi- crobubbles are purely intravascular and therefore allow visualization of the brain vasculature, showing the field of view in the insonated sector (Fig. 2) ${ }^{1,24}$ Acerbi and colleagues described the use of CEUS in conjunction with indocyanine green fluorescence imaging to assess aneurysmal flow and parenchymal perfusion in a distal partially thrombosed aneurysm before and after superficial temporal artery-to-distal middle cerebral artery bypass and efferent vessel aneurysm clipping to induce thrombosis in the aneurysm itself. ${ }^{1}$ The flow in the aneurysm as well as parenchymal perfusion was assessed without exposing the aneurysm as CEUS allows a real-time tomographic section of the surgical field. This application of CEUS allows real-time imaging of the aneurysm as well as the feeding and draining vessels before and after clipping., ${ }^{1,24}$ 

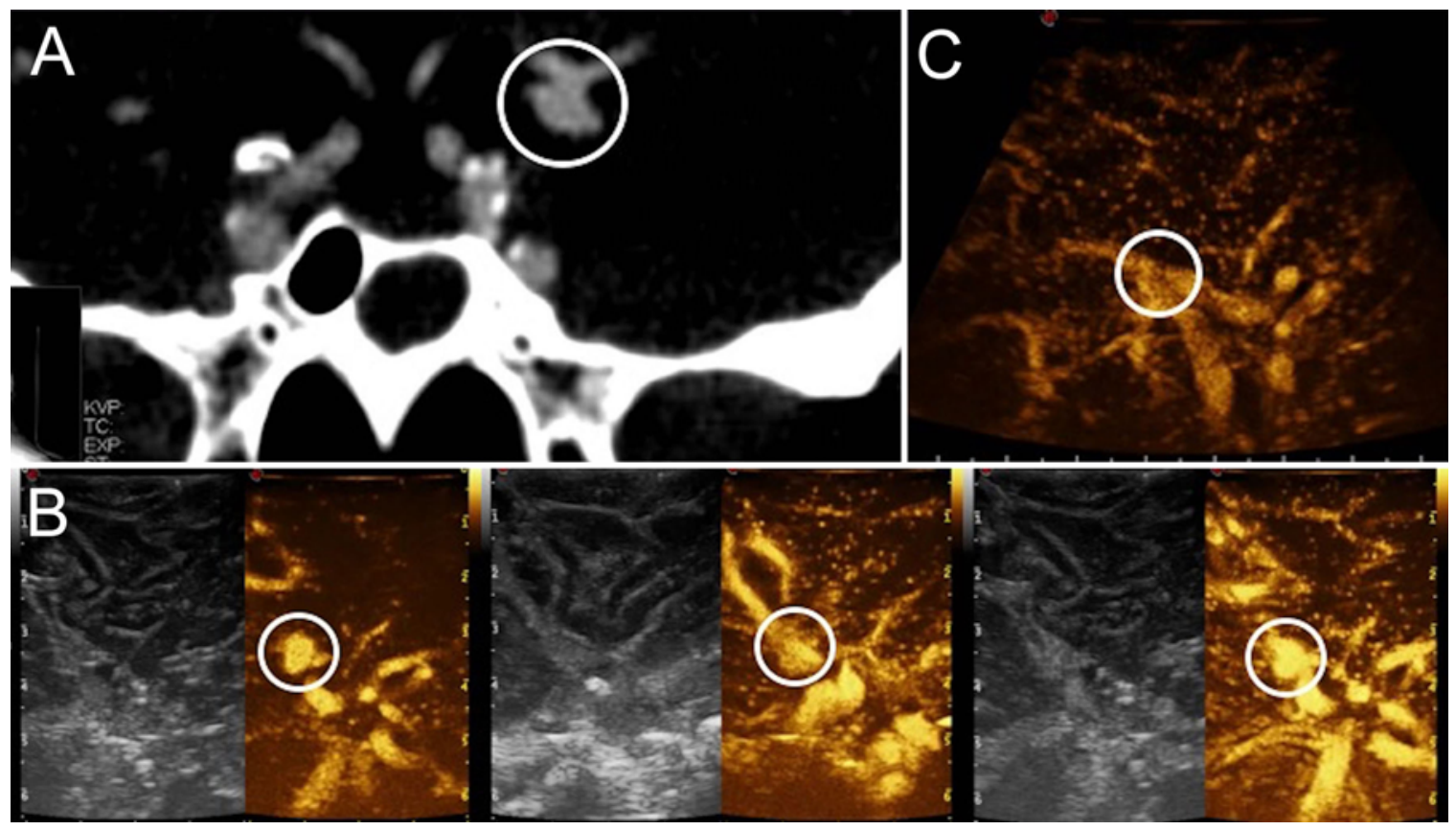

FIG. 2. A: CTA demonstrating an internal carotid artery (ICA) bifurcation aneurysm (circle) on a coronal reconstruction. B: Preoperative CEUS demonstrating three views of the brain parenchyma and ICA bifurcation aneurysm (circles), obtained by tilting the probe via a pterional window at the time of surgery. C: Postclipping CEUS demonstrating occlusion of the ICA aneurysm (circle).

\section{Carotid Visualization}

CEUS has proven to be useful in distinguishing between occlusion and stenosis of the carotid arteries, as well as in identifying plaque ulceration and neovascularization (Fig. 3). ${ }^{4}$ Brinjikji and colleagues discovered that CEUS contrast microbubbles tend to aggregate on ulcerated plaques due to interaction with the highly expressed matrix metalloproteinase-9 and to phagocytosis by activated macrophages within the plaque. ${ }^{4}$ The degree of plaque enhancement on CEUS is associated with the amount of neovascularity and inflammation within the plaque itself and has been shown to suggest greater potential for subsequent cardiovascular events. ${ }^{4}$ Xiong et al. have also reported a correlation between the degree of CEUS enhancement and both the histopathological grade of carotid plaques and serum levels of high-sensitivity C-reactive protein. ${ }^{32}$ This is unsurprising given that neovascularization and macrophage recruitment directly contribute to immunohistochemical grade and that high-sensitivity C-reactive protein is an acute-phase reactant indicative of active inflammation such as that seen in ulcerated plaques. ${ }^{32}$

Ballotta et al. found that CEUS is a safe and effective way to evaluate symptomatic carotid plaques. ${ }^{3}$ They discussed the utility of CEUS in identifying plaques, even those considered to be low-grade, which are at increased risk of rupture. They concluded that the CEUS modality may help to identify those patients in need of surgical intervention. ${ }^{3}$ Additionally, in a recent study on the correlation between MRI and CEUS evaluation of carotid plaques, Shimada and colleagues compared the plaque-tomuscle ratio calculated via MRI and the median brightness of plaque enhancement found using CEUS, which both independently correlate to plaque vulnerability. ${ }^{28}$
They identified a significant association between these values, which suggested diagnostic and prognostic agreement between the two techniques.

\section{Future Applications}

Recent advances in CEUS technology include the addition of targetable ligands to the contrast microbubbles such that the contrast concentrates on a particular area. ${ }^{14} \mathrm{It}$ is also possible to place drugs or DNA plasmids within the microbubbles to administer targeted treatment directly to the area of interest. ${ }^{14}$ This use may also extend to thrombolytic therapies by increasing the effect of recombinant tissue plasminogen activator or by mechanically lysing clots with ultrasound waves. ${ }^{31}$ Sonothrombolysis has already been proven to be both safe and efficacious in treating thromboembolic stroke, with no significant increase in rebleeding rates. ${ }^{31}$

Other areas of potential advancement include validated characterization of various intracranial lesions that could further improve preoperative grading and diagnosis. ${ }^{25}$ Further CEUS evaluation of tumor subcategories could identify universally identifiable patterns of enhancement that would contribute to less invasive diagnosis with increased certainty for the operating surgeon. ${ }^{25}$ More research is also necessary to perfect the use of CEUS to evaluate changes in tumor vascular architecture after resection. ${ }^{16}$ The resection cavity disrupts the preoperative vessel layout and obliterates predetermined surgical landmarks. CEUS may be useful in intraoperatively mapping those landmarks and increasing the rate of complete lesion resection. $^{16}$

Table 1 outlines the findings reported by the series analyzed in this review. 


\section{Guidelines}

According to the EFSUMB guidelines regarding the use of CEUS in evaluating cerebral vessels, the major indication for this modality is to characterize the vascular anatomy and blood flow within pathological intracranial structures to optimize excision. ${ }^{29}$ Intraoperative CEUS allows the surgeon to determine tumor and vascular malformation borders as surgery progresses and also helps to determine the extent of excision before the operation concludes. Combining CEUS with transcranial Doppler ultrasound allows for identification of blood vessels and evaluation of both surrounding brain parenchyma and intravascular flow. Doppler ultrasound alone does not produce sensitive-enough information to determine flow velocity, given its low signal-to-noise ratio. In the setting of traumatic brain injury, CEUS exhibits greater accuracy in distinguishing normal brain parenchyma from injured tissue than B-mode and color Doppler ultrasound. These EFSUMB guidelines are met with strong consensus, despite the paucity of evaluative research..$^{29}$

Despite the efficacy of CEUS, the EFSUMB guidelines indicate the importance of proper operator training for a thorough evaluation. Additionally, care must be taken to ensure the craniotomy is large enough to allow the transducer to appropriately visualize the intracranial anatomy. It should also be noted that static retractors and hemostatic materials cause artifacts during CEUS imaging. It is important to be aware of both the benefits and risks associated with CEUS to avoid causing further damage to patients while attempting to ameliorate the diagnostic and prognostic processes.

\section{Advantages}

Several of the reports on CEUS have provided comparisons with established intraoperative imaging modalities. Digital subtraction angiography (DSA) provides invaluable information on the larger vascular layout surrounding the lesion and can be used to identify minute changes in velocity and direction of blood flow. ${ }^{22}$ However, DSA in the operating theater can be a logistical challenge and also exposes the patient and surgical team to radiation with each use..$^{22}$ Indocyanine green videoangiography is also frequently used to identify vascular malformations and to delineate vascular flow, though vessels can only be visualized if exposed by the surgeon. ${ }^{22}$ Vasculature must abut the pial surface or be directly exposed to the microscope to be fluorescently illuminated., ${ }^{7,8}$ Doppler ultrasound, while it can be used in conjunction with CEUS, is limited by sound wave artifacts and is restricted by the angle of the probe. ${ }^{22}$

The vast majority of patients who undergo CEUS do not experience any adverse reactions (i.e., dizziness, headache, or abdominal pain). ${ }^{5}$ CEUS also provides extensive information even before surgical manipulation without exposing the patient to radiation. ${ }^{8,33}$ Thus, CEUS can be repeated several times intraoperatively to evaluate the vascular anatomy as the surgery progresses. ${ }^{7,2,23}$ This is particularly useful when it becomes necessary to evaluate the extent of tumor excision, by highlighting any residual tumor perfusion after the initial surgical removal., ${ }^{2,5,721}$
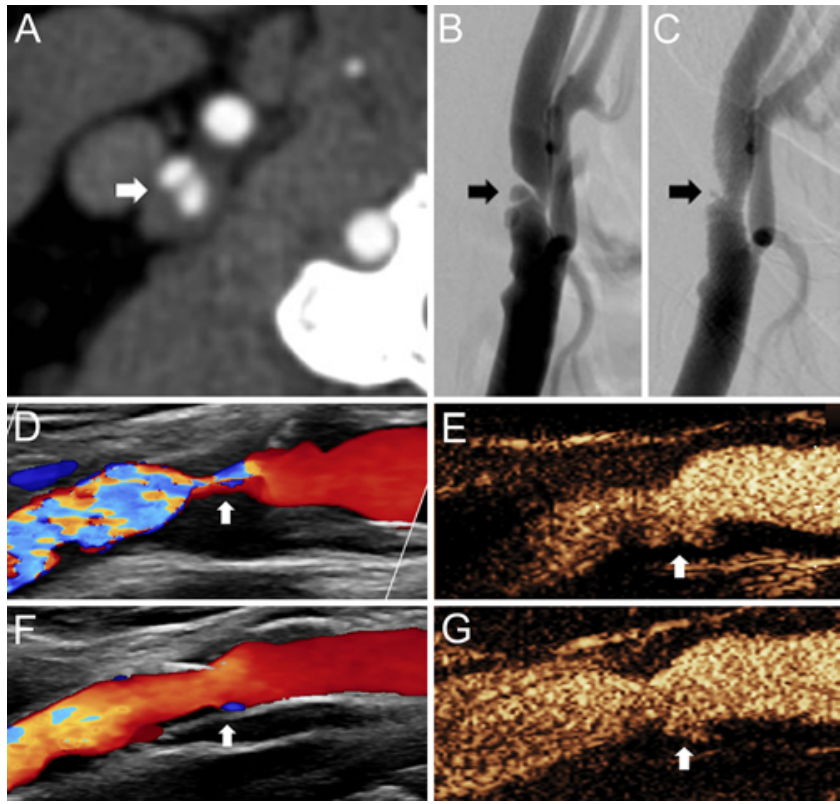

FIG. 3. A: Axial CTA demonstrating plaque ulceration (arrow). B: Lateral right ICA angiogram demonstrating severe stenosis and plaque ulceration (arrow). C: Lateral right ICA angiogram obtained after carotid stent placement, demonstrating improved stenosis and decreased plaque ulceration (arrow). D: Carotid duplex ultrasound demonstrating severe stenosis of the right ICA (arrow). Note that the plaque ulceration is not readily visible. E: CEUS demonstrating severe stenosis of the right ICA with visualization of the ulcerated plaque (arrow). F: Post-carotid stent duplex ultrasound demonstrating significant improvement in the vessel caliber. Note that the residual plaque ulceration is not visible (arrow). G: Post-carotid stent CEUS demonstrating significant improvement in the vessel caliber with visualization of the residual ulcerated plaque (arrow).

The metabolic products of the contrast agent used in this modality are expelled through the lungs rather than the kidneys, as happens with MRI and CT, and can be safely used in patients with chronic kidney disease. ${ }^{28}$

CEUS allows for the visualization of both superficial and deep vascular structures, reducing the need for invasive surgical exposure while also decreasing the overall time of surgery. ${ }^{23,24,33}$ Particularly when guided by prior B-mode ultrasound, CEUS provides a broader view of the anatomical relationship among the lesion of interest, the surrounding parenchyma, and vessels of any diameter and flow velocity. ${ }^{21}$ In most cases, CEUS can clarify borders between the tumor mass and peritumoral edema that are otherwise ill-defined on regular B-mode ultrasound imaging. ${ }^{25}$ While CEUS requires a small craniotomy window that can restrict movement of the probe and limit the field of visualization, it is often not necessary to manipulate the probe at extreme angles..$^{21}$ When such angles are required, surgeons can use transdural insonation of the CEUS contrast to gain a broader view. ${ }^{21}$ Vessel wall integrity can also be determined using this imaging modality after long surgeries that have involved significant vessel manipulation. ${ }^{21}$ Additionally, CEUS has been shown to characterize carotid plaque ulceration better than standard ultrasound. ${ }^{28}$ Often, CEUS is performed in conjunction with Doppler ultrasound to provide information on both the structural anatomy and the type of blood flow visualized. ${ }^{8}$ 
TABLE 1. Overview of articles considered in this review and summaries of their various applications of CEUS in neurosurgery

\begin{tabular}{|c|c|}
\hline Authors \& Year & Summary of Findings \\
\hline \multicolumn{2}{|l|}{ Tumors } \\
\hline Ellegala et al., 2003 & Targeted CEUS contrast agents can detect early tumor angiogenesis \\
\hline Kanno et al., 2005 & $\begin{array}{l}\text { CEUS performed in combination w/ Doppler ultrasonography can be used intraoperatively to } \\
\text { assess vasculature w/in \& surrounding cerebral neoplasms }\end{array}$ \\
\hline He et al., 2008 & $\begin{array}{l}\text { CEUS can delineate tumor \& healthy brain tissue before resection \& is useful in identifying } \\
\text { residual tumor after resection }\end{array}$ \\
\hline Lassau et al., 2011 & $\begin{array}{l}\text { Dynamic CEUS allows quantitative assessment of tumor perfusion by calculating vascular } \\
\text { flow parameters }\end{array}$ \\
\hline Holtel, 2010 & $\begin{array}{l}\text { CEUS can be used to administer drug-containing microbubbles w/in the contrast agent to } \\
\text { allow for targeted therapy }\end{array}$ \\
\hline Prada et al., 2014 & CEUS can be used intraoperatively to characterize the vascularity of intracranial lesions \\
\hline Prada et al., $2015^{21}$ & $\begin{array}{l}\text { CEUS can be used to evaluate skull base tumors \& surrounding vasculature to help determine } \\
\text { appropriate surgical approach }\end{array}$ \\
\hline Ritschel et al., 2015 & CEUS aids in the determination of GBM borders w/ moderate accuracy \\
\hline Arlt et al., 2016 & $\begin{array}{l}\text { CEUS is associated w/ high imaging quality for high-grade gliomas \& can highlight residual } \\
\text { tumor after resection }\end{array}$ \\
\hline Cheng et al., 2016 & $\begin{array}{l}\text { Intraop CEUS can be used to establish the boundary of peritumoral brain edema in glioma } \\
\text { patients }\end{array}$ \\
\hline Ilunga-Mbuyamba et al., 2016 & CEUS can correctly identify small vascular structures surrounding intracranial tumors \\
\hline Prada et al., $2017^{26}$ & CEUS imaging in GBM surgery provides anatomical information comparable to preop MRI \\
\hline Del Bene et al., 2018 & $\begin{array}{l}\text { CEUS can differentiate btwn low- \& high-grade intracranial tumors \& can aid in intraop surgi- } \\
\text { cal decision-making }\end{array}$ \\
\hline Della Pepa et al., 2019 & $\begin{array}{l}\text { CEUS is a safe \& cost-effective intraop imaging modality that allows visualization of unex- } \\
\text { posed anatomical structures \& minimizes degree of surgical disruption of normal paren- } \\
\text { chyma }\end{array}$ \\
\hline \multicolumn{2}{|l|}{ Vascular abnormalities } \\
\hline Hölscher et al., 2007 & $\begin{array}{l}\text { CEUS allows real-time visualization of neurovascular pathologies such as MCA aneurysms \& } \\
\text { AVMs }\end{array}$ \\
\hline Tsivgoulis et al., 2010 & CEUS is safe \& effective for cerebral embolism thrombolysis \\
\hline Prada et al., $2017^{22}$ & $\begin{array}{l}\text { CEUS allows visualization of DAVFs before disrupting the dura, reducing dural damage \& } \\
\text { resulting in more precise fistula excision }\end{array}$ \\
\hline Xu et al., 2017 & $\begin{array}{l}\text { CEUS is useful in determining intraop migration of vessels during resection of deep-seated } \\
\text { AVMs }\end{array}$ \\
\hline Della Pepa et al., 2018 & $\begin{array}{l}\text { CEUS is a simple \& cost-effective imaging modality that allows real-time dynamic visualiza- } \\
\text { tion of DAVFs }\end{array}$ \\
\hline Acerbi et al., 2019 & $\begin{array}{l}\text { CEUS can be used in combination w/ ICGVA to evaluate parenchymal perfusion \& blood flow } \\
\text { after distal outflow occlusion of MCA aneurysms }\end{array}$ \\
\hline \multicolumn{2}{|l|}{ Carotid plaques } \\
\hline Ballotta et al., 2014 & $\begin{array}{l}\text { CEUS is safe \& effective in the treatment \& evaluation of symptomatic low-grade carotid } \\
\text { stenosis w/ an unstable plaque }\end{array}$ \\
\hline Brinjikji et al., 2016 & $\begin{array}{l}\text { CEUS can be used to visualize carotid stenosis plaques \& can help characterize \& predict } \\
\text { plaque vulnerability }\end{array}$ \\
\hline Xiong et al., 2017 & $\begin{array}{l}\text { The degree of CEUS enhancement correlates w/ both the histopathological grade of carotid } \\
\text { plaques \& levels of high-sensitivity C-reactive protein }\end{array}$ \\
\hline Shimada et al., 2018 & $\begin{array}{l}\text { CEUS visualization of carotid plaques can characterize plaque vulnerability to a degree } \\
\text { comparable to preop MRI }\end{array}$ \\
\hline \multicolumn{2}{|l|}{ General } \\
\hline Prada et al., $2015^{24}$ & $\begin{array}{l}\text { CEUS can be used intraoperatively to visualize cerebral vasculature \& evaluate tissue perfu- } \\
\text { sion }\end{array}$ \\
\hline Prada et al., 2018 & $\begin{array}{l}\text { CEUS allows real-time evaluation of both the functional \& anatomical characteristics of } \\
\text { intracranial vasculature }\end{array}$ \\
\hline
\end{tabular}

ICGVA = indocyanine green videoangiography; $\mathrm{MCA}$ = middle cerebral artery. 
Finally, CEUS has proven to be a very safe imaging modality. ${ }^{15,19,20,29}$ The EFSUMB guidelines approve its use in several organ systems such as the endocrine, lymphatic, gastrointestinal, and genitourinary systems, among others. ${ }^{29}$ The microbubbles are excreted via the lung and the liver, which makes CEUS a safe and useful technique in patients with kidney disease. ${ }^{30}$ CEUS also does not utilize iodine or ionizing radiation and has a rate of contrastinduced anaphylaxis of $0.001 \% .9,20 \mathrm{~A}$ recent article on the use of CEUS by Dietrich et al. reports no cardiotoxicity, hepatotoxicity, or nephrotoxicity associated with the use of the microbubble contrast agents.9 These authors also reported a lower incidence of adverse side effects with microbubble contrast agents than with radiographic contrast agents and a comparable rate of adverse effects with MRI contrast. ${ }^{9}$

\section{Limitations}

While CEUS is largely a cost-effective, safe, and informative procedure, there are notable contraindications and limitations to this imaging modality. Administration of the microbubble contrast agent is contraindicated in those with heart disease and those who previously experienced an acute allergic reaction to the contrast. ${ }^{4}$ This technique also requires specific user training, and there is a high risk of interobserver variability in the evaluation of the imaging results. ${ }^{4,8,21,24}$

While a trained surgeon can use CEUS to visualize both deep and superficial structures, the operator is only able to view a portion of the lesion at a given time and can only observe the anatomy in a $2 \mathrm{D}$ plane. ${ }^{8,25,33}$ Particularly when using an embolizing agent, several planes must be assessed. The embolizing agent can obscure information beneath the area of administration, requiring additional imaging planes for complete evaluation of the vascular structures? ${ }^{7}$ However, it is possible to perform multiple sweeps with the ultrasound probe and combine the collected data using an algorithm, which produces a 3D view of the entire lesion. ${ }^{25,33}$ This integration of information from multiple imaging planes can produce a model of the intracranial anatomy that can provide surgeons with more complete information regarding lesion anatomy and blood flow characteristics that were previously unattainable. ${ }^{23}$

Given the inconsistency of flow within tumor neovessels at any time, CEUS may not accurately reflect the general extent of perfusion within a tumor. ${ }^{10,18}$ CEUS may also overestimate tumor size when targeted to markers of angiogenesis. ${ }^{10}$ However, this sensitivity to vascular progression may be useful in identifying those lesions with increased metastatic predilection. ${ }^{10}$ Ultrasound waves gain intensity as they pass through the contrast agent, causing vessels further away from the probe to appear deceptively brighter. ${ }^{4}$ Alternately, calcifications and hemostatic substances exhibit high levels of contrast enhancement that may reflect the sound waves and inhibit proper visualization of deeper structures. ${ }^{21}$ Though CEUS was particularly useful in elucidating the architecture of highly vascular tumors, it exhibited limited utility in some lower-grade gliomas and malignant lymphomas, displaying weak enhancement compared to that demonstrated with preoperative CT and MRI. ${ }^{17}$

\section{Conclusions}

CEUS has proven to be a useful imaging modality across the spectrum of neurosurgical disease. It is a safe, cost-effective procedure that can provide invaluable information to neurosurgeons at any stage of surgery. CEUS lends itself to lesion diagnosis, tumor grading, vascular mapping, planning of surgical approaches, intraoperative evaluation, and identification of residual tumor as well as targeted delivery of medicines. Future research and application of this technology will undoubtedly uncover many more advantages and uses for the treatment of neurosurgical disease.

\section{References}

1. Acerbi F, Prada F, Vetrano IG, Falco J, Faragò G, Ferroli P, et al: Indocyanine green and contrast-enhanced ultrasound videoangiography: a synergistic approach for real-time verification of distal revascularization and aneurysm occlusion in a complex distal middle cerebral artery aneurysm. World Neurosurg 125:277-284, 2019

2. Arlt F, Chalopin C, Müns A, Meixensberger J, Lindner D: Intraoperative 3D contrast-enhanced ultrasound (CEUS): a prospective study of 50 patients with brain tumours. Acta Neurochir (Wien) 158:685-694, 2016

3. Ballotta E, Angelini A, Mazzalai F, Piatto G, Toniato A, Baracchini C: Carotid endarterectomy for symptomatic lowgrade carotid stenosis. J Vasc Surg 59:25-31, 2014

4. Brinjikji W, Huston J III, Rabinstein AA, Kim GM, Lerman A, Lanzino G: Contemporary carotid imaging: from degree of stenosis to plaque vulnerability. J Neurosurg 124:27-42, 2016

5. Cheng LG, He W, Zhang HX, Song Q, Ning B, Li HZ, et al: Intraoperative contrast enhanced ultrasound evaluates the grade of glioma. Biomed Res Int 2016:2643862, 2016

6. Del Bene M, Perin A, Casali C, Legnani F, Saladino A, Mattei L, et al: Advanced ultrasound imaging in glioma surgery: beyond gray-scale B-mode. Front Oncol 8:576, 2018

7. Della Pepa GM, Marchese E, Pedicelli A, Olivi A, Ricciardi L, Rapisarda A, et al: Contrast-enhanced ultrasonography and color Doppler: guided intraoperative embolization of intracranial highly vascularized tumors. World Neurosurg 128:547-555, 2019

8. Della Pepa GM, Sabatino G, Sturiale CL, Marchese E, Puca A, Olivi A, et al: Integration of real-time intraoperative contrast-enhanced ultrasound and color Doppler ultrasound in the surgical treatment of spinal cord dural arteriovenous fistulas. World Neurosurg 112:138-142, 2018

9. Dietrich CF, Averkiou M, Nielsen MB, Barr RG, Burns PN, Calliada F, et al: How to perform contrast-enhanced ultrasound (CEUS). Ultrasound Int Open 4:E2-E15, 2018

10. Ellegala DB, Leong-Poi H, Carpenter JE, Klibanov AL, Kaul $\mathrm{S}$, Shaffrey ME, et al: Imaging tumor angiogenesis with contrast ultrasound and microbubbles targeted to $\alpha(v) \beta 3$. Circulation 108:336-341, 2003

11. Greis C: Ultrasound contrast agents as markers of vascularity and microcirculation. Clin Hemorheol Microcirc 43:1-9, 2009

12. He W, Jiang XQ, Wang S, Zhang MZ, Zhao JZ, Liu HZ, et al: Intraoperative contrast-enhanced ultrasound for brain tumors. Clin Imaging 32:419-424, 2008

13. Hölscher T, Ozgur B, Singel S, Wilkening WG, Mattrey RF, Sang H: Intraoperative ultrasound using phase inversion harmonic imaging: first experiences. Neurosurgery 60 (4 Suppl 2):382-387, 2007

14. Holtel MR: Emerging technology in head and neck ultrasonography. Otolaryngol Clin North Am 43:1267-1274, 2010 
15. Huang L, Zhou K, Zhang J, Ma Y, Yang W, Ran L, et al: Efficacy and safety of high-intensity focused ultrasound ablation for hepatocellular carcinoma by changing the acoustic environment: microbubble contrast agent (SonoVue) and transcatheter arterial chemoembolization. Int J Hyperthermia 36:244-252, 2019

16. Ilunga-Mbuyamba E, Avina-Cervantes JG, Lindner D, CruzAceves I, Arlt F, Chalopin C: Vascular structure identification in intraoperative 3D contrast-enhanced ultrasound data. Sensors (Basel) 16:E497, 2016

17. Kanno H, Ozawa Y, Sakata K, Sato H, Tanabe Y, Shimizu N, et al: Intraoperative power Doppler ultrasonography with a contrast-enhancing agent for intracranial tumors. J Neurosurg 102:295-301, 2005

18. Lassau N, Chami L, Chebil M, Benatsou B, Bidault S, Girard E, et al: Dynamic contrast-enhanced ultrasonography (DCEUS) and anti-angiogenic treatments. Discov Med 11:18-24, 2011

19. Main ML, Ryan AC, Davis TE, Albano MP, Kusnetzky LL, Hibberd M: Acute mortality in hospitalized patients undergoing echocardiography with and without an ultrasound contrast agent (multicenter registry results in 4,300,966 consecutive patients). Am J Cardiol 102:1742-1746, 2008

20. Piscaglia F, Bolondi L, Italian Society for Ultrasound in Medicine and Biology (SIUMB) Study Group on Ultrasound Contrast Agents: The safety of Sonovue in abdominal applications: retrospective analysis of 23188 investigations. Ultrasound Med Biol 32:1369-1375, 2006

21. Prada F, Del Bene M, Casali C, Saladino A, Legnani FG, Perin A, et al: Intraoperative navigated angiosonography for skull base tumor surgery. World Neurosurg 84:1699-1707, 2015

22. Prada F, Del Bene M, Faragò G, DiMeco F: Spinal dural arteriovenous fistula: Is there a role for intraoperative contrastenhanced ultrasound? World Neurosurg 100:712.e15-712. e18, 2017

23. Prada F, Del Bene M, Mauri G, Lamperti M, Vailati D, Richetta C, et al: Dynamic assessment of venous anatomy and function in neurosurgery with real-time intraoperative multimodal ultrasound: technical note. Neurosurg Focus 45(1):E6, 2018

24. Prada F, Del Bene M, Saini M, Ferroli P, DiMeco F: Intraoperative cerebral angiosonography with ultrasound contrast agents: how I do it. Acta Neurochir (Wien) 157:1025-1029, 2015

25. Prada F, Perin A, Martegani A, Aiani L, Solbiati L, Lamperti $\mathrm{M}$, et al: Intraoperative contrast-enhanced ultrasound for brain tumor surgery. Neurosurgery 74:542-552, 2014

26. Prada F, Vitale V, Del Bene M, Boffano C, Sconfienza LM, Pinzi V, et al: Contrast-enhanced MR imaging versus contrast-enhanced US: a comparison in glioblastoma surgery by using intraoperative fusion imaging. Radiology 285:242249, 2017
27. Ritschel K, Pechlivanis I, Winter S: Brain tumor classification on intraoperative contrast-enhanced ultrasound. Int J CARS 10:531-540, 2015

28. Shimada H, Ogata T, Takano K, Abe H, Higashi T, Yamashita T, et al: Evaluation of the time-dependent changes and the vulnerability of carotid plaques using contrast-enhanced carotid ultrasonography. J Stroke Cerebrovasc Dis 27:321325, 2018

29. Sidhu PS, Cantisani V, Dietrich CF, Gilja OH, Saftoiu A, Bartels E, et al: The EFSUMB guidelines and recommendations for the clinical practice of contrast-enhanced ultrasound (CEUS) in non-hepatic applications: update 2017 (long version). Ultraschall Med 39:e2-e44, 2018

30. Tedesco G, Sarno A, Rizzo G, Grecchi A, Testa I, Giannotti $\mathrm{G}$, et al: Clinical use of contrast-enhanced ultrasound beyond the liver: a focus on renal, splenic, and pancreatic applications. Ultrasonography 38:278-288, 2019

31. Tsivgoulis G, Eggers J, Ribo M, Perren F, Saqqur M, Rubiera M, et al: Safety and efficacy of ultrasound-enhanced thrombolysis: a comprehensive review and meta-analysis of randomized and nonrandomized studies. Stroke 41:280-287, 2010

32. Xiong L, Sun WJ, Cai HY, Yang Y, Zhu J, Zhao BW: Correlation of enhancement degree on contrast-enhanced ultrasound with histopathology of carotid plaques and serum high sensitive C-reactive protein levels in patients undergoing carotid endarterectomy. J Huazhong Univ Sci Technolog Med Sci 37:425-428, 2017

33. Xu H, Qin Z, Xu M, Chen C, Zhang J, Chen X: Clinical experience with intraoperative ultrasonographic image in microsurgical resection of cerebral arteriovenous malformations. World Neurosurg 97:93-97, 2017

\section{Disclosures}

The authors report no conflict of interest concerning the materials or methods used in this study or the findings specified in this paper.

\section{Author Contributions}

Conception and design: Park, Prada. Acquisition of data: Kearns. Analysis and interpretation of data: Kearns, Chadwell, Chandler, Kiernan, Prada, Kalani. Drafting the article: Park, Kearns, Sokolowski, Prada. Critically revising the article: Park, Kearns, Sokolowski, Prada. Reviewed submitted version of manuscript: all authors. Approved the final version of the manuscript on behalf of all authors: Park. Administrative/technical/material support: Park.

\section{Correspondence}

Min S. Park: University of Virginia Health System, Charlottesville, VA. mp2tq@hscmail.mcc.virginia.edu. 